\title{
Combination of Inverted ILM Flap Technique and Subretinal Fluid Application Technique for Treatment of Chronic, Persistent and Large Macular Holes
}

\author{
Lyubomyr M. Lytvynchuk (1) - Andrii Ruban - Carsten Meyer · \\ Knut Stieger · Andrzej Grzybowski · Gisbert Richard
}

Received: May 1, 2021 / Accepted: June 5, 2021 / Published online: July 6, 2021

(c) The Author(s) 2021

\section{ABSTRACT}

Introduction: The choice of surgical treatment for chronic, persistent and large full-thickness macular holes (FTMH) continues to be undefined and challenging, as some of these cases remain refractory to the treatment. We report the efficacy of combination of inverted internal

Lyubomyr M. Lytvynchuk and Andrii Ruban contributed equally to this work.

Supplementary Information The online version contains supplementary material available at https://doi. org/10.1007/s40123-021-00361-2.

L. M. Lytvynchuk $(\varangle) \cdot$ K. Stieger

Department of Ophthalmology, Eye Clinic, Justus-

Liebig-University Giessen, University Hospital

Giessen and Marburg GmbH, Campus Giessen,

Friedrichstrasse 18, 35392 Giessen, Germany

e-mail: Lyubomyr.Lytvynchuk@augen.med.uni-

giessen.de

K. Stieger

e-mail: Knut.Stieger@uniklinikum-giessen.de

L. M. Lytvynchuk

Karl Landsteiner Institute for Retinal Research and Imaging, Juchgasse 25, 1030 Vienna, Austria

A. Ruban

Center of Clinical Ophthalmology, Peremogy Ave.

42, Kyiv 03057, Ukraine

e-mail: ruban33@gmail.com limiting membrane flap technique (IILMFT) and subretinal application of the fluid (SR fluid application) technique for treatment of refractory FTMHs.

Methods: Nine patients (nine eyes) were enrolled into this retrospective non-randomized exploratory consecutive case series study. All patients were diagnosed with chronic, persistent or large FTMH and were treated with a combination of IILMFT and SR fluid application technique. The following outcome parameters were analysed during 1- and 6-month follow-up visits: anatomical FTMH closure rate on spectral domain optical coherence tomography (SDOCT), best-corrected visual acuity (BCVA),

\section{Meyer}

Augenärzte Kammanneye, Bahnhofstrasse 3, 7270

Davos, Switzerland

e-mail: meyer_eye@yahoo.com

\section{A. Grzybowski}

Department of Ophthalmology, University of Warmia and Mazury, Zolnierska 18, 10-561 Olsztyn, Poland

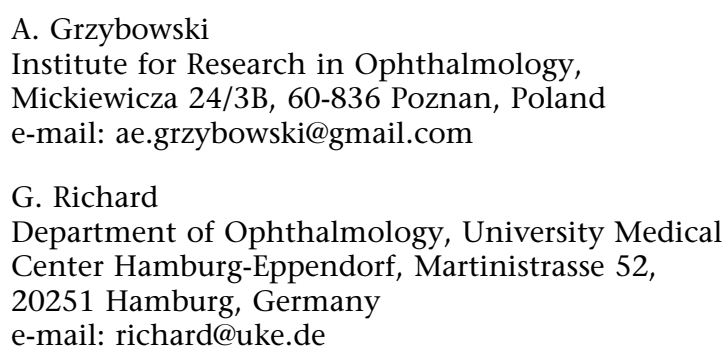

G. Richard

Department of Ophthalmology, University Medical Center Hamburg-Eppendorf, Martinistrasse 52, 20251 Hamburg, Germany

e-mail: richard@uke.de 
degree of postoperative retinal displacement.

Results: The mean preoperative diameter of FTMH was $542.0 \mu \mathrm{m}$ (range 154-1930 $\mu \mathrm{m}$ ). Final closure of FTMH was achieved in nine of nine cases $(100 \%)$. In one case a second operation was required because of postoperative rhegmatogenous retinal detachment. The mean BCVA after the FTMH closure increased from 1.0 $\log M A R \quad(0.7-1.3)$ to $0.4 \log \mathrm{MAR} \quad(0.2-$ $0.8 \log$ MAR $)(W=2.67 ; p=0.008)$. A positive correlation was revealed between preoperative BCVA and axial length ( $\rho=0.67, p=0.048)$, between preoperative BCVA and duration of the symptoms $(\rho=0.818, p=0.007)$, as well as between postoperative BCVA at 1-month follow-up and BCVA at 6-month follow-up $(\rho=0.821, p=0.007)$.

Conclusion: Combination of IILMFT with SR fluid application technique for refractory FTMH surgery appears to be effective and safe. Improvement of anatomical and visual outcomes after the single surgery benefits from and is ensured by the advantages of both novel surgical approaches.

Keywords: Hydraulic centripetal macular displacement technique; Inverted ILM flap; Macular hole closure; Pars plana vitrectomy; Persistent macular hole; Retinal adhesion; Subretinal fluid application

\section{Key Summary Points}

Why carry out this study?

Surgical treatment of chronic, persistent and large full-thickness macular holes (FTMH) remains challenging as the pathogenesis is still unclear.

Modern surgical techniques, such as inverted internal limiting membrane flap technique (IILMFT) and subretinal fluid application technique (SR fluid application), allow surgeons to increase the rate of anatomical closure, but the failure rate still exists.

\section{What was learned from the study?}

The use of a novel combination of two techniques, IILMFT and SR fluid application, for treatment of chronic, persistent and large FTMH during the same surgical procedure proved to be effective and safe with a final closure rate of $100 \%$ (9/9 cases).

The study results imply that the combined effects of both techniques, namely centripetal mobilization of the retina and induction of glial proliferation under the ILM flap, can complement the shortcomings of both, leading to final complete $\mathrm{MH}$ closure.

Application of temporal internal limiting membrane flap with local ILM peeling nasally to FTMH for subretinal injection can minimize the iatrogenic intraoperative impact from ILM removal.

\section{DIGITAL FEATURES}

This article is published with digital features, including a summary slide, to facilitate understanding of the article. To view digital features for this article go to https://doi.org/10.6084/ m9.figshare.14731776.

\section{INTRODUCTION}

Since the initial report by Kelly and Wendel, vitrectomy has become the standard approach in the treatment of idiopathic full-thickness macular hole (FTMH), which includes pars plana vitrectomy (PPV), removal of posterior cortical vitreous, epiretinal membrane (ERM) and the internal limiting membrane (ILM), complete fluid-gas exchange, and face-down positioning postoperatively [1]. The efficacy of modern minimally invasive surgical approaches in macular hole (MH) surgery exceeds $90 \%$. However, chronic, persistent, recurrent and large MHs have long been known to have the 
worst prognosis with regard to the conventional surgical technique $[2,3]$. While a number of unique surgical approaches have been proposed, none of them has became the gold standard.

Several surgical adjuncts that aim to reduce tractional forces and promote $\mathrm{MH}$ closure have been proposed to treat large and chronic FTMH [4-6]. The inverted ILM flap technique (IILMFT), which was reported by Michalewska et al. [7], was developed with the aim of enhancing closure of complicated cases of FTMH, including those with high myopia. A good anatomical and functional outcome was obtained in patients receiving either the classic or the temporal inverted ILM flap technique, or even free ILM flap. It was suggested that in IILMFT, the ILM acts as a scaffold for glial cell migration and proliferation, and the air or gas provides only additional stabilization.

Oliver and Wojcik first reported the novel techniques of macular detachment with subretinal injection of balanced salt solution (BSS) to treat persistent idiopathic $\mathrm{MH}$ [8]. The authors hypothesized that subretinal BSS reduces the tension and stiffness of the retina, thus making it more compliant and allowing the edges of the hole to reapproximate. In 2017, we reported the efficacy of hydraulic centripetal macular displacement (HCMD) in treatment of posttraumatic FTMH in six cases [9]. This technique achieved anatomical $\mathrm{MH}$ closure in $85.7 \%$ of cases and the size of $\mathrm{MH}$ significantly decreased in $14.3 \%$. In 2020 Meyer et al. reported the primary outcomes of the application of subretinal (SR) fluid to close refractory FTMH in 41 cases (APOSTEL Study) [10]. The study results demonstrated secondary $\mathrm{MH}$ closure in $85.36 \%$ at 6 weeks after the surgery with improvement of best-corrected visual acuity (BCVA).

The efficacy of intraoperative spectral domain optical coherence tomography (iSDOCT) imaging for macular surgery, including FTMH, was proved and reported in many studies [11-14]. Recently, we reported the ability of iSD-OCT to reveal additional and useful information regarding tissue behaviour (retinal stretching, dynamics of the $\mathrm{MH}$ base) during IILMFT [14].

The aim of this study was to analyse the efficacy of a modified surgical approach to treat chronic, persistent and large FTMH, which combines inverted ILM flap technique and SR fluid application during one surgical procedure. We hypothesized that the combination of two novel techniques will improve the rate of $\mathrm{MH}$ closure.

\section{METHODS}

\section{Ethics Statement}

This retrospective observational exploratory study was performed at the Department of Ophthalmology, Justus Liebig University, University Hospital Giessen and Marburg $\mathrm{GmbH}$, Campus Giessen (Giessen, Germany) and at the Center of Clinical Ophthalmology (Kyiv, Ukraine) with the aim of analysing the efficacy of the combination of two novel surgical techniques in treatment of chronic, persistent and large MHs. The study protocol was approved by the Ethics Committee of Justus Liebig University, Department of Medicine (Giessen, Germany) with protocol number AZ $55 / 21$. The study matched the tenets of the Declaration of Helsinki. In all cases, a signed consent was obtained from the patients.

\section{Patients}

Nine patients (nine eyes) with FTMH were operated on using the combined surgical technique. Data were analysed retrospectively. The main inclusion criteria were (1) presence of chronic, persistent or large FTMH; (2) use of combined surgical technique (inverted ILM flap technique and SR application technique); (3) postoperative follow-up for a minimum of 6 months. The main exclusion criteria were (1) the absence of follow-up in postoperative period, and (2) the use of only one of the aforementioned surgical techniques. 


\section{Examination Technique}

A standard and full ophthalmic examination was performed pre- and postoperatively in every case. It included BCVA with ETDRS charts (with computation to logMAR chart), Goldmann tonometry, slit-lamp examination and indirect fundus ophthalmoscopy. The presence of FTMH was examined and documented with digital imaging using a fundus camera (CX-1 (Canon, Tokyo, Japan) and Clarus 500 (Carl Zeiss Meditech, Oberkochen, Germany)) and SD-OCT systems (Spectralis ${ }^{\circledR}$ (Heidelberg Engineering GmbH, Heidelberg, Germany), RTVue (Optovue, Fremont, CA, USA) and SS OCT Angio ${ }^{\mathrm{TM}}$ (Topcon Corporation, Tokyo, Japan). The imaging diagnostic was performed preoperatively and at 1, 3 and 6 months after the surgery. MH size was measured according to the protocol of the International Vitreomacular Traction Study (IVTS) Group [15] using minimal width of the FTMH, which was measured at the narrowest point of the $\mathrm{MH}-$ minimum linear diameter (MLD). This distance was measured with the caliper function on the SD-OCT system by drawing a line parallel to the retinal pigment epithelium (RPE) layer [15].

The extent of macular displacement was revealed and documented by overlapping of pre- and postoperative red-free fundus images, considering optic disc (OD) with major vessels within $1500 \mu \mathrm{m}$ around the OD to be the reference points during overlap. Anatomical closure of $\mathrm{MH}$ and improvement of the BCVA were the major criteria to evaluate the efficacy of the combined surgical technique.

With regard to its pathogenesis, the FTMHs were classified into idiopathic, myopic and traumatic. The diagnosis of myopic $\mathrm{MH}$ was established on eyes with axial length (AL) $>27.00 \mathrm{~mm}$. The types of full-thickness MHs were defined as follows: chronic $\mathrm{MH}$ - with duration > 6 months; persistent $\mathrm{MH}-$ failed to close after the previous surgery/ies; large $\mathrm{MH}-$ with size $>400 \mu \mathrm{m}$ (in accordance with the protocol of the International Vitreomacular Traction Study Group).

Pre- and postoperative presence of central scotoma was examined in all cases with the

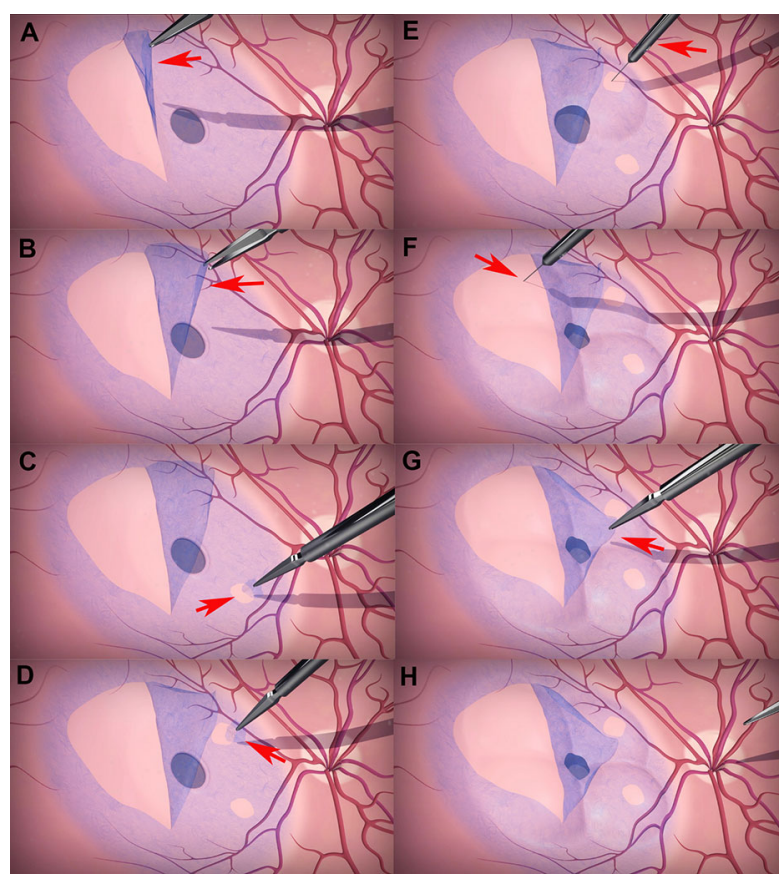

Fig. 1 Combination of temporal ILM flap technique with subretinal application of the fluid for treatment of chronic, persistent and large FTMH. Schematic depiction of the surgical steps: A, B creation of the temporal ILM flap (red arrows); C, D focal removal of ILM nasally to the $\mathrm{MH}$ (red arrows); E, F subretinal application of the fluid in four quadrants (red arrows); covering of the MH with temporal ILM flap at the end of the surgery (red arrow)

Amsler test. Every case was analysed with regard to intra- and postoperative complication.

\section{Surgical Procedure}

A standard three-port PPV was performed in all cases using a vitrectomy system EVA (D.O.R.C., Zuidland, Netherlands) or Constellation ${ }^{\circledR V i-}$ sion System (Alcon, Fort Worth, TX, USA) with a non-contact viewing system and a contact macula lens. The surgical steps of the combination of two techniques are schematically presented on Fig. 1 and Video S1 (see Video S1 in the electronic supplementary material for details). Posterior vitreous detachment and its removal anteriorly to the equator were done in cases with chronic idiopathic MH (two cases). In all cases the staining of the ILM was performed with Membrane Blue Dual (D.O.R.C., Zuidland, 
Netherlands). Then, a "classic", temporal inverted, pedicle ILM flap or free ILM flap was created. In the case of temporal ILM flap, small parts of ILM were removed additionally in two areas between fovea and OD in order to facilitate straightforward SR fluid application (Video S1) [16]. In the case of free ILM flap, a perfluorocarbon liquid (Deca-Vit Syringe, Vitreq, MG Vierpolders, Netherlands) was used to stabilize and ensure proper position of the ILM flap. In some cases, ILM removal was extended to a 3-4-OD-diameter area around the FTMH within the intended square of iatrogenic macular detachment. SR fluid application was performed with SR injections of balanced salt solution (BSS ${ }^{\circledR}$, Alcon Laboratories, Inc., Fort Worth, TX, USA). SR PolyTip cannula 25/38 gauge (MedOne Surgical, Inc. Sarasota, FL, USA) was connected through a Luer lock adapter to the syringe filled with BSS. SR fluid application was accomplished in four quadrants. Puncture retinotomies were placed within a $2-4$-OD-diameter area from the FTMH. During application of SR fluid, the infusion pressure was lowered to $15 \mathrm{mmHg}$ by changing settings of the vitrectomy system. SR injection of BBS was controlled visually and was stopped at the point when the retinal bleb reached the edges of FTMH and BSS leaked through the $\mathrm{MH}$. Then, the detached retina in the perifoveal area was gently massaged in the centripetal direction using a backflush cannula (D.O.R.C., Zuidland, Netherlands) to bring the hole edges closer to each other. Afterwards, the $\mathrm{MH}$ was covered with ILM flap and air-fluid exchange was performed with thoroughgoing drainage of SR fluid (Video S1). The type of endotamponade was determined during the surgery and included sterile air $100 \%$, a gas-air mixture of sulfur hexafluoride $\left(\mathrm{SF}_{6} 20 \%\right)$ or silicon oil $5000 \mathrm{cSt}$. All patients were requested to stay in a facedown position for $24 \mathrm{~h}$.

\section{iSD-OCT Imaging}

Intraoperative SD-OCT assisted approach with Rescan ${ }^{\circledR} 700$ (Carl Zeiss Meditech, Oberkochen, Germany) was applied in three cases (surgeries done by LL). The Rescan ${ }^{\circledR} 700$ possess the following technical characteristics: spectral domain OCT engine with scanning speed $27,000 \mathrm{~A}$-scans per second and wavelength of $840 \mathrm{~nm}$; refresh rate $5-50 \mathrm{~Hz}$; axial resolution $3.5-5.5 \mu \mathrm{m}$. The scan depth varied from 2 to $5.6 \mathrm{~mm}$ with an adjustable scan length (range 3-18 mm). iSD-OCT data was analysed during and after the surgery.

\section{Graphical Animation of Surgical Technique}

Schematic depiction of inverted temporal ILM flap technique with SR application of the fluid for treatment of chronic, persistent and large FTMH was performed using graphical animation software Autodesk Maya (Alias Systems Corporation, Toronto, Canada) by Mr. Mikhail Akinyaev (Kyiv, Ukraine).

\section{Statistical Methods}

Obtained data were analysed statistically with the following methods: Wilcoxon signed-rank test, the Spearman rank-order correlation coefficient, interquartile range (IQR) for descriptive statistics, frequency tables, correlation matrices, Student's $t$ test. For statistical analysis a software STATISTICA 10 (StatSoft, Inc., OK, USA) was used. Statistical significance was considered when the $p$ value was 0.05 or less.

\section{RESULTS}

\section{Patients}

Nine Caucasian patients with a diagnosis of chronic, persistent and/or large FTMH were included in this study cohort: seven women (77.78\%) and two men (22.22\%). The description of pathological mechanism and type of FTMH is presented in Table 1 . Mean age of the patients was 59 years (range 27-69 years old). Six right eyes $(66.67 \%)$ and three left eyes $(33.33 \%)$ were treated. Mean axial length was $24.3 \mathrm{~mm}$ (range $22.03-30.15 \mathrm{~mm}$ ) with mean spherical equivalent - 3.0 diopters (D) (range form -12.5 to $+2.5 \mathrm{D}$ ). The mean size (MLD) 


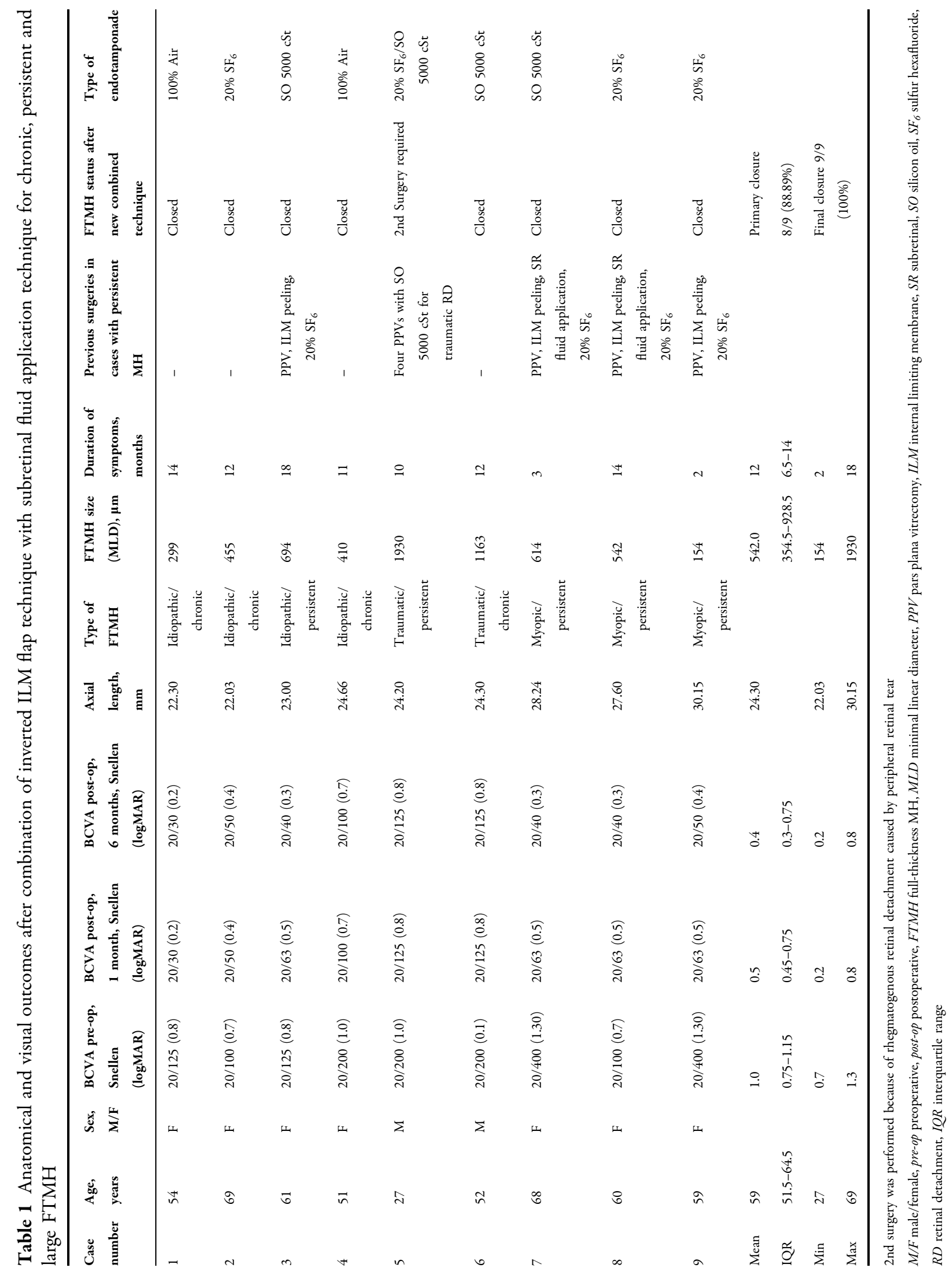




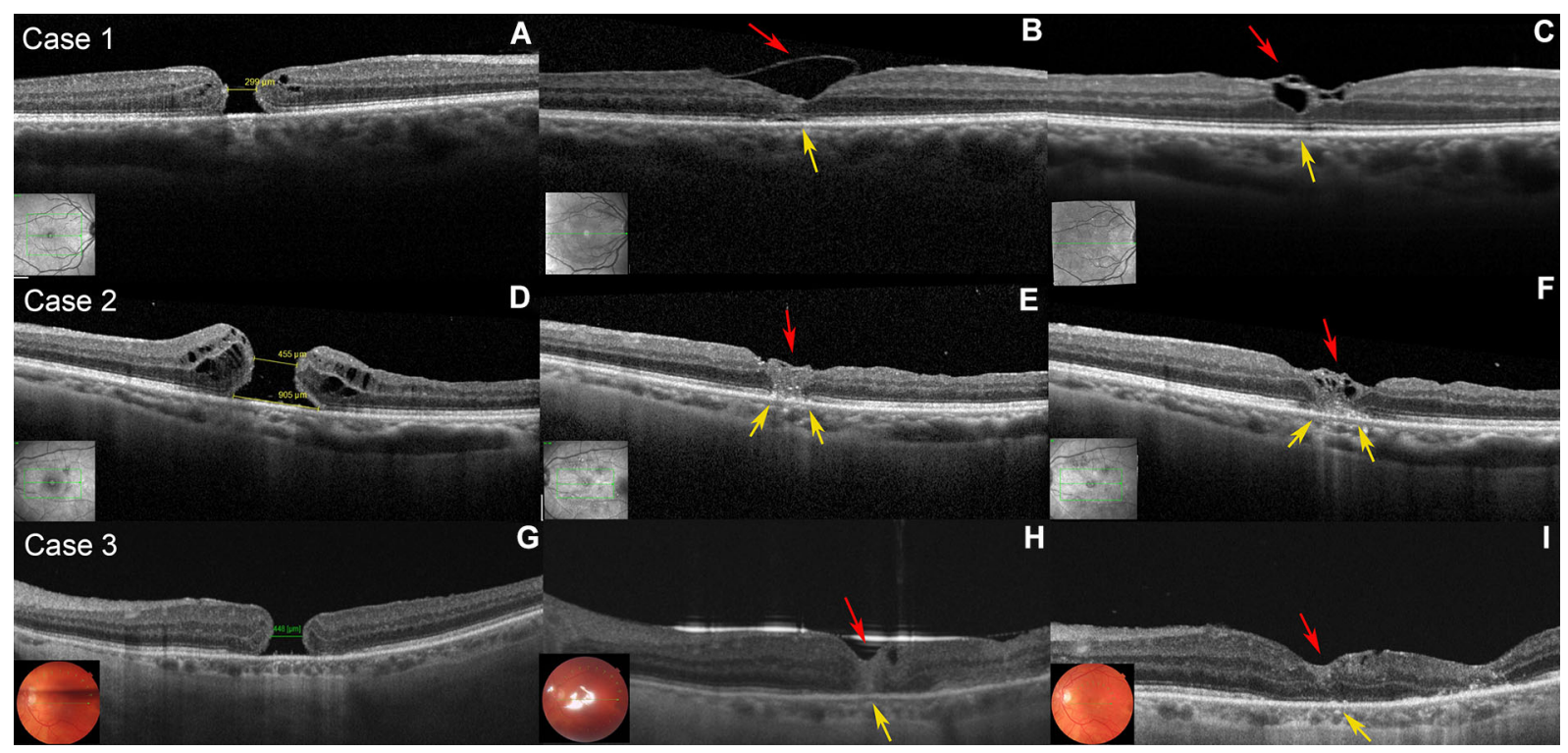

Fig. 2 Preoperative (A, D, G), 1-month postoperative (B, $\mathbf{E}, \mathbf{H})$ and 3-month postoperative $(\mathbf{C}, \mathbf{F}, \mathbf{I})$ OCT scans of the foveal area of case 1, 2 and 3. Case 1: primary apposition of chronic idiopathic $\mathrm{MH}$ edges (B, yellow arrow) with remaining ILM flap (B, C, red arrows). Ongoing restoration of the foveal anatomy ( $\mathbf{C}$, yellow arrow). Case 2: primary closure of persistent operated idiopathic FTMH with ILM flap (E, F, red arrows) without apposition of the FTMH edges (E, F yellow

of FTMH was $542.0 \mu \mathrm{m}$ (range 154-1930 $\mu \mathrm{m}$ ). The mean duration of FTMH was 12 months (range 2-18 months) (Table 1).

\section{Surgery}

Surgical steps of the combination of inverted temporal ILM flap technique with SR fluid application for FTMH treatment are depicted schematically on Fig. 1 and Video S1. All surgeries were performed by two experienced vitreoretinal surgeons (three cases by LL and six cases by AR). Pars plana vitrectomy was performed with combined 23/27-gauge (27-gauge trocar was used for infusion) in two cases, and with 25-gauge in seven cases. In all cases, a combination of SR fluid application technique with centripetal displacement of the macula using silicon-tipped cannula was combined with inverted ILM flap technique: "classic" inverted ILM flap technique was used in two arrows). Case 3: persistent operated idiopathic FTMH after the first surgery, which decreased in size $(\mathbf{G})$. Almost complete apposition of FTMH edges after the second surgery with $\mathrm{SO}$ endotamponade ( $\mathbf{H}$ yellow arrow) with remnants of the ILM flap on top of the fovea $(\mathbf{H}$ red arrow). A complete apposition of $\mathrm{MH}$ edges (yellow arrow) with ILM remnants (red arrow) remained after SO was removed (I)

cases (22.2\%), inverted pedicle ILM flap technique was used in three cases (33.3\%), temporal ILM flap in three cases (33.3\%) and free ILM flap in one case (11.2\%) (Videos S2 and S3). For intraocular endotamponade, $100 \%$ sterile air was used in two cases, a gas-air mixture of sulfur hexafluoride $\left(\mathrm{SF}_{6} 20 \%\right)$ in four cases and silicon oil $5000 \mathrm{cSt}$ in three cases. In two traumatic/ persistent $\mathrm{MH}$ cases treated with SO (cases 6 and 7) there was a large minimal linear diameter (930 and $1163 \mu \mathrm{m}$ ) and strong SR adhesion revealed during SR fluid application (indication for SO endotamponade). In one case of myopic/ persistent $\mathrm{MH}$, SO was used to prevent the displacement of the free ILM flap.

\section{Anatomical Results}

$\mathrm{MH}$ closure was achieved in eight cases $(88.89 \%)$ after the primary surgery with novel technique (Figs. 2, 3, 4, 5). In one case (11.11\%) 


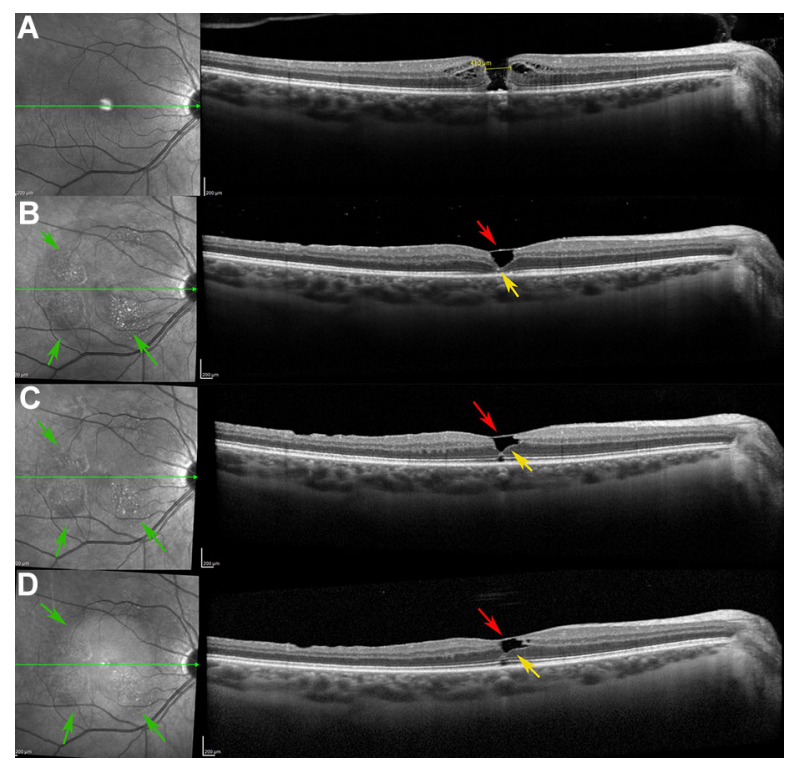

Fig. 3 Preoperative (A), early postoperative (B) and late postoperative $(\mathbf{C}, \mathbf{D})$ OCT scans of the foveal area of case 4. Primary apposition of the base of chronic idiopathic MH (B, yellow arrow) with ILM flap on top of the fovea (B, red arrow). Ongoing filling of the fovea with retinal cells (C, D, yellow arrows) under the ILM flap (C, D, red arrow). Hyperreflective zones on red-free photos in areas of SR fluid application (B, green arrows), which slowly disappear in late follow-ups (C, D, green arrows)

a complete macular closure was achieved after the second surgery, which was indicated because of rhegmatogenous retinal detachment caused by peripheral retinal break (Fig. 4, Table 1). Final MH closure rate was 100\% (9/9 cases).

A gradual and ongoing filling of the partially apposed FTMH edges and under the inverted ILM flap with retinal tissue was revealed in OCT in case 1, 4, 6, 7, 8 and 9 (Figs. 2B, C, 3C, D yellow arrows).

In cases 2 and 5, the closure of FTMH was achieved mainly by filling of the $\mathrm{MH}$ with inverted and free ILM flap, respectively (Fig. 2E, F red arrows, Fig. 4G red arrow). Additionally, a certain degree of centripetal displacement of macular tissue was noticed, which in our opinion reinforced $\mathrm{MH}$ closure.

In all cases, SR fluid application caused retinal displacement, which was documented on red-free images by overlaying a pre- and postoperative fundus images. Retinal displacement after combination of IILMFT and SR fluid application technique in case 3 is depicted in Fig. 5.

\section{Functional Results}

Preoperative mean BCVA in this study group was 1.0 logMAR (range 1.3-0.7 logMAR) (Table 1). Postoperative mean BCVA at 1-month follow-up was 0.5 logMAR (range 0.8-$0.2 \log$ MAR $) \quad(W=2.69 ; p=0.007) \quad($ Table 1$)$. Postoperative mean BCVA at 6-month followup was 0.4 $\log$ MAR (range 0.8-0.2 $\log \mathrm{MAR}$ ) $(W=2.67 ; p=0.008)$ (see Table S1 in the electronic supplementary material for details). A strong positive correlation was revealed between preoperative BCVA and axial length ( $\rho=0.67, \quad p=0.048)$, between preoperative BCVA and duration of the symptoms $(\rho=0.818, p=0.007)$, as well as between postoperative BCVA at 1-month follow-up and BCVA at 6-month follow-up $(\rho=0.821$, $p=0.007$ ) (see Table $\mathrm{S} 2$ in the electronic supplementary material for details). All patients before the surgery reported central scotoma using the Amsler test. At 6-month follow-up, there was an absence of central scotoma in the Amsler test in all patients.

\section{Complications}

In all cases there were no intra- or postoperative complications related to combination of IILMFT and SR fluid application technique. In one case with traumatic FTMH (case 5), a rhegmatogenous retinal detachment developed in early postoperative period, which was caused by peripheral retinal tear (Fig. 4D, E). Even though the ILM flap was partially preserved and partially lost due to retinal detachment (Fig. 4E red arrow), the size of the FTMH remained smaller compared to preoperative condition (Fig. 4C yellow arrows). This complication necessitated second pars plana vitrectomy with additional placement of free ILM flap and SO endotamponade in order to ensure $\mathrm{MH}$ closure (Fig. 4F yellow arrow, G red arrow).

Postoperative hyperreflective zones were documented in macula on red-free images in 


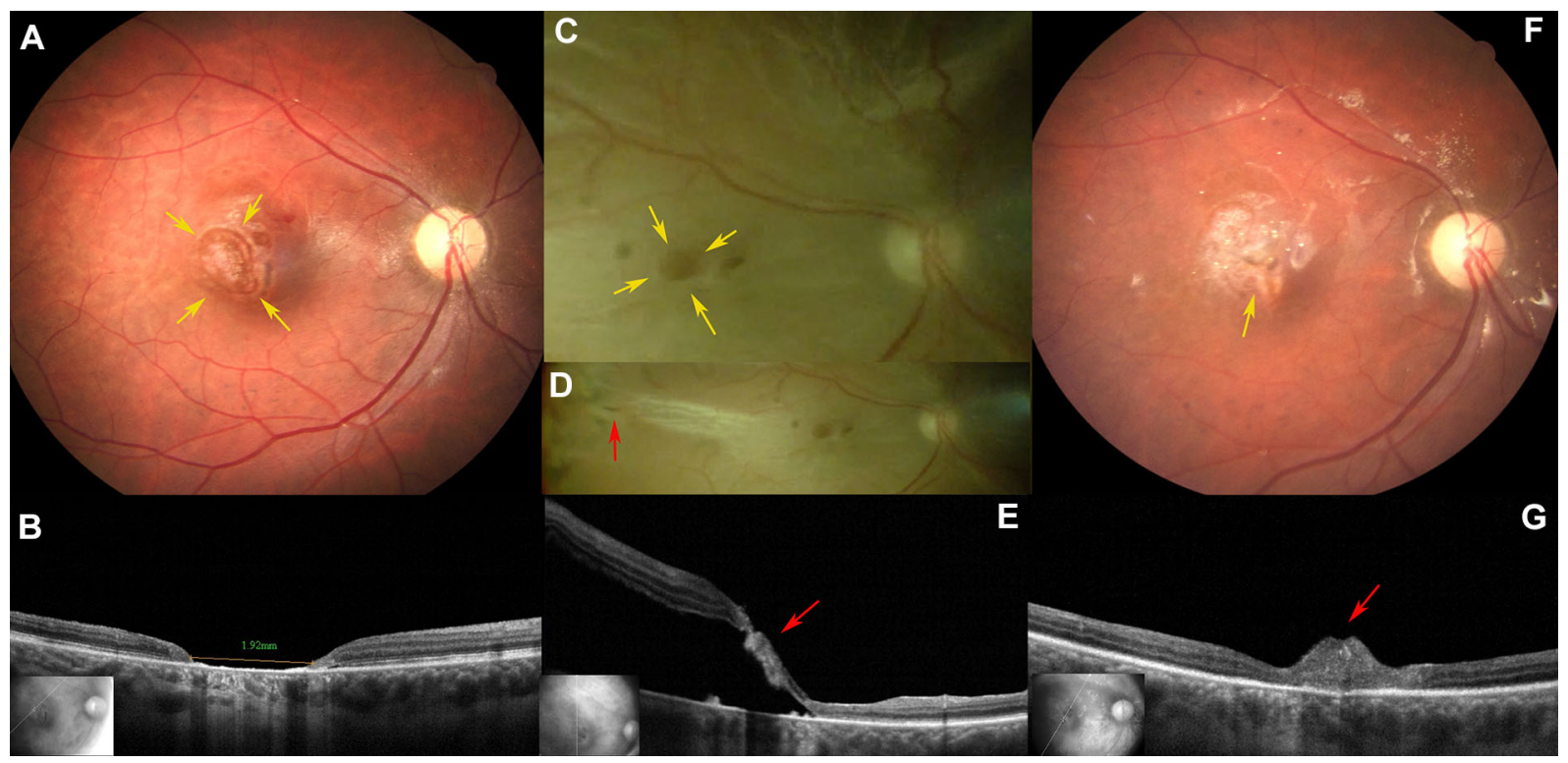

Fig. 4 Preoperative photo and OCT scan of posttraumatic large $\mathrm{MH}$ in case 5 (A, B). A Yellow arrows indicate the edges of $\mathrm{MH}$. Development of postoperative rhegmatogenous retinal detachment $(\mathbf{C}-\mathbf{E})$ caused by peripheral retinal tear (D, red arrow). The size of $\mathrm{MH}$ has decreased (C, yellow arrows). E ILM flap partially remained on the top of $\mathrm{MH}$ (red arrow). Closure of $\mathrm{MH}$ after reoperation due to retinal detachment ( $\mathbf{F}$, yellow arrow). An additional positioning of the free ILM flap was used to achieve a complete closure of $\mathrm{MH}$ (G, red arrow) areas of SR fluid application in three cases $(33.3 \%)$, which however gradually resolved within 6-month follow-up and did not cause any complains or positive Amsler test results (Fig. 3B-D green arrows).

\section{Analysis of iSD-OCT Imaging}

The iSD-OCT assisted pars plana vitrectomy was used in three cases (cases 1, 2, 4). The real-time iSD-OCT approach allowed for more controlled performance of every step of the surgery on the macula. It allowed one to control the application of SR fluid, showing that there were no unintentional subchoroidal injections of the fluid (Fig. 6A-C yellow arrow). Additionally, iSD-OCT assisted during inversion of the ILM flap by ensuring its correct position during and at the end of the surgery (Fig. 6A-C red arrows, Video S2).

\section{DISCUSSION}

Primary anatomical closure of chronic, persistent and large FTMH remains challenging and may be affected by various pre-, intra- and postoperative factors, such as advanced stage of FTMH, its duration, MH size, various types of manoeuvres with ILM and poor compliance of the patient with postoperative positioning $[9-11,17,18]$.

Our study focused on the application of a novel combination of two surgical techniques during one surgical procedure for treatment of chronic, persistent and large FTMH. Application of SR fluid in the macular area together with inverted ILM flap technique demonstrated its efficacy regarding anatomical closure and improvement of the BCVA in our cohort with closure after primary surgery in $8 / 9$ cases $(88.89 \%)$ and with final closure in $9 / 9$ cases (100\%) (Table 1). 


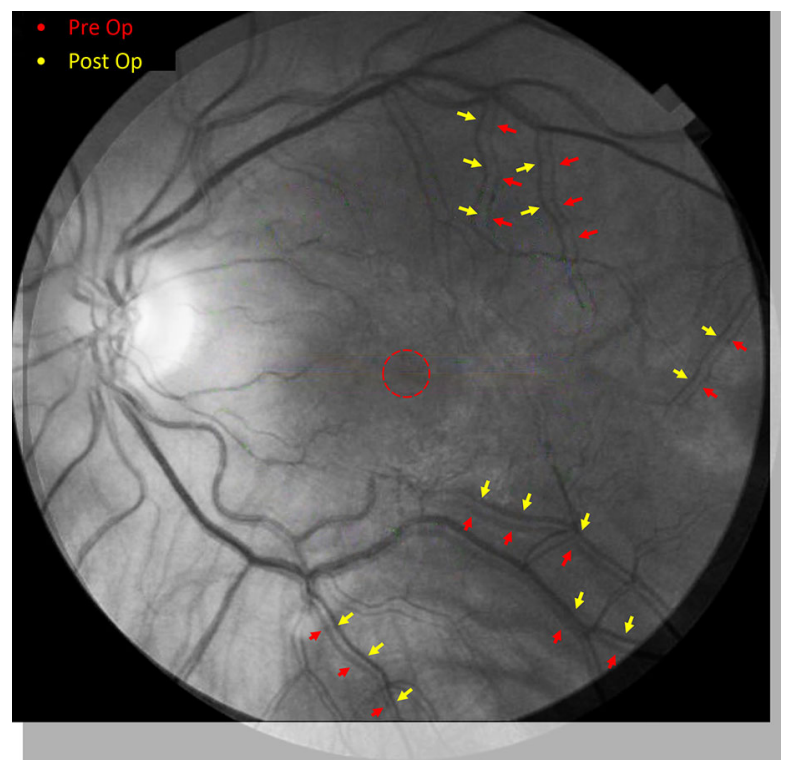

Fig. 5 Degree of retinal displacement in macular area after MH surgery with free ILM flap technique and SR fluid application for treatment of posttraumatic MH. Retinal displacement is depicted by superimposing of preoperative and postoperative red-free digital fundus photographs (case 4). The position of the macular vessels and dimension of the $\mathrm{MH}$ preoperatively are indicated with red arrows and dashed line, respectively. The position of displaced macular vessels postoperatively is indicated with yellow arrows

The inverted ILM flap technique first described by Michalewska et al. showed its efficacy in treatment of large idiopathic and myopic FTMHs [7, 19, 20]. It involves placing of small remnants of peeled ILM that are left attached to the hole margins on the $\mathrm{MH}$ upside down. It is thought that the presence of the ILM flap acts as a scaffold for glial cell proliferation within the hole, which subsequently stimulates some migration of photoreceptor cells toward the fovea [21]. The authors reported that the technique increased their rate of complete FTMH closure to $98 \%$ with improved visual acuity for large idiopathic MHs $(>400 \mu \mathrm{m})$ compared with an $88 \%$ closure rate using conventional vitrectomy and ILM peeling. A number of study groups have since reported similar success in closure rates and improved visual outcomes with this technique [22-28]. In our cohort during the surgeries, we used different types of inverted ILM flap technique including classic, temporal, inverted pedicle and free ILM flap technique. Rizzo et al. reported that vitrectomy performed with IILMFT appeared to be more effective than the conventional ILM peeling technique to treat large idiopathic $\mathrm{MHs}$ $(>400 \mu \mathrm{m})$ and myopic MHs [22]. Success rate of $95.6 \%$ was achieved in the IILMFT group in comparison to $78.6 \%$ in the ILM peeling group. Among the patients with an $\mathrm{AL}>26 \mathrm{~mm}$, a success rate was $88.4 \%$ in the IILMFT group and $38.9 \%$ in the ILM peeling group.

Michalewska et al. later introduced the modified temporal inverted ILM flap technique, where the iatrogenic trauma associated with ILM peeling was minimized to the temporal area [20]. Shin et al. described a modified technique with a single-layered flap of the ILM [27]. Andrew et al. reported a $100 \%$ closure rate in a single procedure for chronic large FTMH with significant visual acuity improvement in $95.8 \%$ after modified technique with ILM flap, which enhanced retention of the ILM flap on the macular surface [28].

The exact cause of failure of primary $\mathrm{MH}$ surgery is still uncertain. Hillenkamp et al. suggested that a key point in closing the hole is a centripetal movement of retinal tissue in the absence of an adhesion between the hole margin and the underlying RPE (as seen in the holes "with cuff"), with the subsequent healing response mediated by the proliferation of glial cells [29]. The fact that the hole height, included in the calculation for three major indexes (MH index, tractional hole index, and hole form factor), is an important positive prognostic predictor also confirms this statement [29-32].

Implementing this concept in 2011, Oliver and Wojcik first reported the macular detachment technique with SR injection of balanced salt solution (BSS) to treat persistent idiopathic $\mathrm{MH}$ [8]. In recent years, several studies have been published using this technique in the surgery of primary, persistent and traumatic MHs [9, 10, 33-36]. Despite the different names of this technique used by the authors, the key principle remains unchanged-injection of fluid under the retina. The hypothesis is that BSS injected into the SR space releases the MH edges from the underlying RPE and reduces 


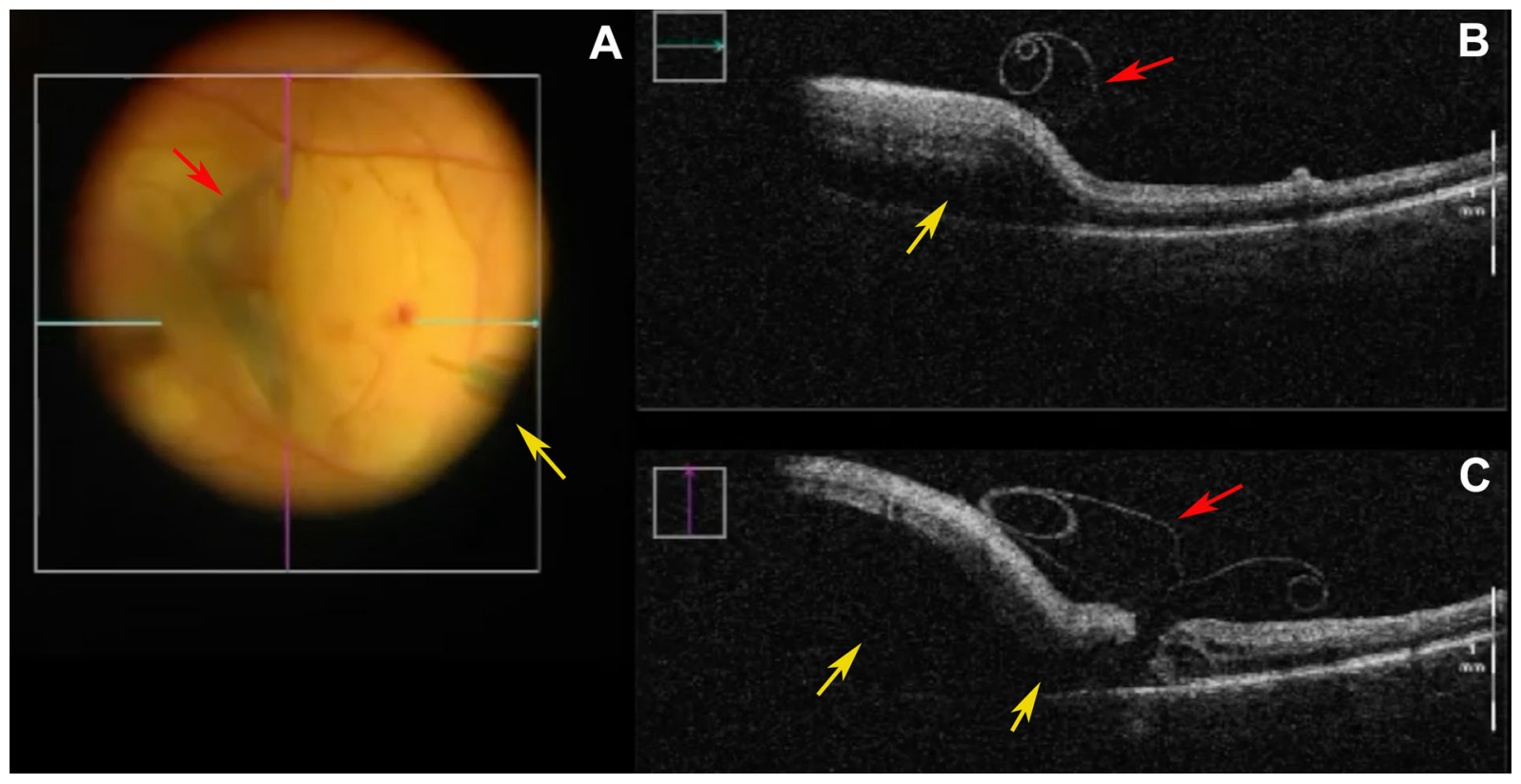

Fig. 6 iSD-OCT cross-hair scan during combination of IILMFT and SR fluid application technique in case 1. A Intraoperative view shows inverted temporal ILM flap over the $\mathrm{MH}$ (red arrow) and moment of subretinal

tension and stiffness intrinsic to the retina, allowing the edges of the hole to reapproximate. This makes the retina more compliant and allows the edges of the $\mathrm{MH}$ to reapproximate [8-10].

Szigiato et al. reported the results of ten patients with persistent or recurrent idiopathic MHs after treatment with similar to macular detachment technique [33]. In two cases, they additionally massaged the macular retina with a diamond-dusted scraper teasing the edges of the $\mathrm{MH}$ with Eckardt forceps. After an air or air-gas endotamponade was performed, all patients maintained a face-down position for 1 week. In nine of the 10 patients (90\%) a closure of the $\mathrm{MH}$ postoperatively was observed up to the 6-month follow-up with subjective improvement of visual acuity.

Wong published a retrospective series of 16 patients with MHs greater than $650 \mu \mathrm{m}$ after treatment with standard PPV and ILM peeling using the same technique-retina expansion technique for MH apposition (RETMA) [34]. Successful MH closure after the procedure was application of the fluid with subretinal cannula (yellow arrow). B, C Extent of iatrogenic detachment of retina after application of subretinal fluid (yellow arrows). Inverted temporal ILM flap over the $\mathrm{MH}$ (red arrows)

achieved in $83 \%(14 / 16)$ of eyes. At 12-month follow-up, no worsening in visual acuity was reported, and improvement in visual acuity was noted in 14 of 16 eyes.

Chakrabarti et al. [35] reported results of posterior pole detachment technique in seven eyes of seven patients with large idiopathic, traumatic and post-rhegmatogenous retinal detachment (RRD) full-thickness $\mathrm{MH}$. The mean preoperative hole size was $587 \mu \mathrm{m}$. The authors used a shorter-acting gas $\left(\mathrm{SF}_{6}\right)$, did not manipulate or massage the retina, and patients were not routinely postured. Anatomical success was achieved in $6 / 7$ eyes (85.7\%).

Claes described the retinal redistribution technique to treat very large primary $\mathrm{MHs}$ $(800-1500 \mu \mathrm{m})$, as well as persistent MHs [36]. Six eyes with hole size ranging from 800 to $1500 \mu \mathrm{m}$ were treated accordingly. This technique included standard vitrectomy, ILM peeling, SR fluid application, injection of perfluorodecalin liquid (PFCL) bubble in order to prevent leakage of the subretinal BSS via the hole, and injection of $1000 \mathrm{cSt}$ silicone oil with 
the complete drainage of the BSS. The silicone oil tamponade was removed after 6 weeks. In our study we used SO endotamponade in three cases, which was removed within 2 months after the surgery.

In 2017 we presented a similar approach and showed that the hydraulic centripetal macular displacement technique is also an effective and safe method to treat large traumatic $\mathrm{MH}$. Combined with SR fibrous scarring dissection and centripetal massaging of the retina, this technique resulted in a closure rate of $85.7 \%$ [9].

Despite the indisputable advantages of the techniques listed above, several shortcomings should be noted. One of the problems after application of IILMFT for large FTMH is the "flap closure type" of $\mathrm{MH}$, which might be observed in $14-16 \%$ of cases [21]. Several authors have reported the efficacy of IILMFT with insertion of ILM into $\mathrm{MH}$ as the initial treatment of $\mathrm{MH}$ with retinal detachment in high myopia [37-39]. With regard to closing MHs in high myopia, we hypothesize that the IILMFT due to the filling of the MH with proliferating cells might have more advantages than the retinal relocation technique alone. In case 2, we observed the aforementioned type of closure of chronic idiopathic $\mathrm{MH}$ when inverted ILM flap filled the space between the edges of $\mathrm{MH}$, thus promoting glial reaction (Fig. 2E, F). This case demonstrates that SR fluid application did not cause MH closure, but it was an inverted ILM flap that ensured the anatomical closure of FTMH. In contrast, in cases 1 and 4, MH closure was achieved by apposition of $\mathrm{MH}$ base, while the inverted ILM flap supported the further healing process (Figs. 2 and 3).

Yun et al. [40] reported that chronic MHs often have tight adhesions of the photoreceptor layer of the retina to the underlying RPE. Meyer et al. [10] advocated that remaining retinal adhesion in $\mathrm{MH}$ can explain the failure rate after the surgery of persistent MHs with application of SR fluid as well. That is why we suggest that combination of two effective techniques (SR fluid application and inverted ILM flap technique) may increase the closure rate after surgery of chronic, persistent and large FTMHs.

In order to reduce trauma to Müller cells nasally to $\mathrm{MH}$, we performed SR injection of saline with a non-penetrative technique through the areas with local ILM removal nasally to $\mathrm{MH}$, as proposed by Okanouchi et al. [16]. To the best of our knowledge, it is the first description of application of this approach during SR fluid application technique for treatment of large FTMH. Together with temporal ILM flap technique, local ILM removal nasally from FTMH for SR injection can minimize the iatrogenic intraoperative impact of ILM removal and SR fluid application.

Kumagai et al. [41] studied the displacement of capillaries in the macular area after pars plana vitrectomy with ILM peeling and showed that conventional ILM peeling causes the displacements of capillaries in a centripetal manner in the early postoperative period. In 2019, Shiono et al. reported the comparative results of retinal displacement after temporal ILM peeling and $360^{\circ}$ ILM peeling for $\mathrm{MH}$ treatment [42]. The authors concluded that displacement of the retinal vessel was significantly less 1 week postoperatively in a group with temporal ILM peeling, stating that hemi-temporal ILM peeling might be preferable as less displacement of the retina is safer for the macula compared to $360^{\circ}$ ILM peeling. In our cohort a centripetal displacement of retinal tissue in the macular was one of the main goals, which was achieved through SR fluid application (Fig. 5). Application of hemi-temporal or $360^{\circ}$ ILM peeling can hypothetically increase to some extent centripetal displacement due to release of the superficial tractional forces of ILM.

The condition of iatrogenic induced central retinal detachment requires a proper endotamponade, which shall ensure retinal reattachment and a stable position of the inverted ILM flap. In 2019, Schawkat et al. [43] studied the influence of postoperative posture on the development of retinal folds with retinal shift after macula-off retinal detachment surgery, which can be visualized with fundus autofluorescence images (FAF). The authors concluded that postoperative positioning of the patients with log roll (type of face-down position) did not significantly influence the postoperative retinal slippage rate after retinal detachment surgery with gas endotamponades. However, intraoperative use of heavy liquids may be 
associated with lower risk of retinal slippage. In our cohort we used heavy liquids intraoperatively in 5/9 cases with the aim of stabilizing inverted ILM flap and face-down position for $24 \mathrm{~h}$ in $9 / 9$ patients. There were no cases of retinal slippage on FAF in our cohort. Additionally, we suppose that endotamponade with silicon oil could support the stability of free ILM flap in large FTMHs in the postoperative period, while gas-air mixture can be used in cases with chronic and persistent FTMHs.

Efficacy of application of iSD-OCT real-time imaging during inverted ILM flap technique was studied earlier by Lytvynchuk et al. [14]. In our cohort the use of iSD-OCT approach in three cases allowed us to control SR fluid application as well as correct placement of inverted ILM flap on the top of MH (Fig. 6, Video S2). With iSDOCT we could also ensure the correct position of the ILM flap at the end of the surgery after gas-air endotamponade was performed.

Limitations of this study include the absence of a control group. Nonetheless, we could compare our data with the data of our previous study, where posttraumatic large FTMHs were treated with SR application of BSS only with a closure rate of $85.7 \%$, compared to the current study with a final closure rate of $100 \%$. The small number of patients operated on and the limited follow-up period are other limitations of this study. However, it is difficult to recruit a larger number of patients with chronic, persistent or larger FTMH on one site, as this is rather a rare diagnosis. The use of different types of inverted ILM flap technique could be considered as another study limitation, which makes the intraoperative surgical conditions in all cases unequal. On the other hand, the use of modified techniques with ILM flap was required in certain cases of persistent FTMH, as these patients had already been operated on with ILM removal. The lack of analysis of the influence of the newly proposed technique on the ellipsoid zone and external limiting membrane on OCT during the postoperative period is a further limitation of this study. It could be solved by planning future studies with larger numbers of patients.

In summary, we suggest that combination of two independent techniques will allow one to increase synergistically their effectiveness and reduce their disadvantages and failure rates. Future studies of efficacy of the proposed novel combined surgical techniques should be planned and their outcomes should be analysed and compared to the outcomes of conventional approaches with the aim of developing an optimal strategy for treatment of refractory FTMHs.

\section{CONCLUSION}

Surgical treatment of chronic, persistent and large FTMH remains difficult. Up till now there is no consensus as to which technique should be used first. There are a number of newly invented techniques which allow one to increase the closure rate, including inverted ILM flap technique and SR fluid application technique. However, failures of $\mathrm{MH}$ closure have been reported. To the best of our knowledge, this study is the first report about the combination of two novel techniques (IILMFT and SR fluid application) as treatment for most refractory types of MHs, such as chronic, persistent and large FTMH with a final closure rate of $100 \%$ (9/9 cases). The study results show that the use of two techniques during one surgical procedure appeared to be safe. The synergy of the anatomical outcomes of two techniques, namely centripetal mobilization of the retina and proliferation of glial cells under the ILM flap, seems to cover the failure rates of each other leading to complete $\mathrm{MH}$ closure. Nevertheless, the efficacy of this combined approach should be studied further.

\section{ACKNOWLEDGEMENTS}

Funding. This publication was funded by Justus Liebig University (Giessen, Germany). No Rapid Service Fee was received for the publication of this article.

Authorship. All named authors meet the International Committee of Medical Journal Editors (ICMJE) criteria for authorship for this 
article, take responsibility for the integrity of the work as a whole, and have given their approval for this version to be published.

Author Contributions. Conceptualization: Lyubomyr Lytvynchuk, Andrii Ruban; Methodology: Lyubomyr Lytvynchuk, Andrii Ruban; Formal analysis and investigation: Lyubomyr Lytvynchuk, Andrii Ruban; Writing-original draft preparation: Lyubomyr Lytvynchuk, Andrii Ruban; Writing-review and editing: Lyubomyr Lytvynchuk, Andrii Ruban, Carsten Meyer, Knut Stieger, Andrzej Grzybowski, Gisbert Richard; Funding acquisition: Knut Stieger; Supervision: Carsten Meyer, Knut Stieger, Andrzej Grzybowski, Gisbert Richard. All authors read and approved the final manuscript.

Disclosures. Lyubomyr Lytvynchuk, Andrii Ruban, Carsten Meyer, Knut Stieger, Andrzej Grzybowski and Gisbert Richard declare that they have no conflict of interest.

Compliance with Ethics Guidelines. The study protocol was approved by the Ethics Committee of Justus Liebig University, Department of Medicine (Giessen, Germany) with protocol number AZ 55/21. This study was performed in accordance with the Helsinki Declaration of 1964, and its later amendments. All subjects provided informed consent to participate in the study. This manuscript does not contain any identifying information of the patients. This article is based on previously conducted studies and does not contain any new studies with human participants or animals performed by any of the authors.

Data Availability. Data generated or analysed during this study are partially included in this published article/as supplementary information files. The datasets generated during and/ or analysed during the current study are available from the corresponding author on reasonable request.

Thanking Patient Participants. We would like to thank all study participants for their involvement in the study.
Open Access. This article is licensed under a Creative Commons Attribution-NonCommercial 4.0 International License, which permits any non-commercial use, sharing, adaptation, distribution and reproduction in any medium or format, as long as you give appropriate credit to the original author(s) and the source, provide a link to the Creative Commons licence, and indicate if changes were made. The images or other third party material in this article are included in the article's Creative Commons licence, unless indicated otherwise in a credit line to the material. If material is not included in the article's Creative Commons licence and your intended use is not permitted by statutory regulation or exceeds the permitted use, you will need to obtain permission directly from the copyright holder. To view a copy of this licence, visit http://creativecommons.org/licenses/by$\mathrm{nc} / 4.0 /$.

\section{REFERENCES}

1. Kelly NE, Wendel RT. Vitreous surgery for idiopathic macular holes. Results of a pilot study. Arch Ophthalmol. 1991;109:654-9.

2. Ullrich S, Haritoglou C, Gass C, Schaumberger M, Ulbig MW, Kampik A. Macular hole size as a prognostic factor in macular hole surgery. Br J Ophthalmol. 2002;86:390-3.

3. Thompson JT, Sjaarda RN, Lansing MB. The results of vitreous surgery for chronic macular holes. Retina. 1997;17:493-501.

4. Ezra E, Gregor ZJ. Surgery for idiopathic full-thickness macular hole: two-year results of a randomized clinical trial com- paring natural history, vitrectomy, and vitrectomy plus autologous serum: Moorfields Macular Hole Study Group Report no. 1. Arch Ophthalmol. 2004;122:224-36.

5. Kumar A, Tinwala SI, Gogia V, Sehra SV. Tapping of macular hole edges: the outcomes of a novel technique for large macular holes. Asia Pac J Ophthalmol (Phila). 2013;2:305-9.

6. Charles S, Randolph JC, Neekhra A, Salisbury CD, Littlejohn N, Calzada JI. Arcuate retinotomy for the repair of large macular holes. Ophthalmic Surg Lasers Imaging Retina. 2013;44:69-72. 
7. Michalewska Z, Michalewski J, Adelman RA, Nawrocki J. Inverted internal limiting membrane flap technique for large macular holes. Ophthalmology. 2010;117:2018-25.

8. Oliver A, Wojcik E. Macular detachment for treatment of persistent macular hole. Ophthalmic Surg Laser Imag. 2011;42:6.

9. Ruban A, Lytvynchuk L, Zolnikova A, Richard G. Efficiency of the hydraulic centripetal macular displacement technique in the treatment of traumatic full-thickness macular holes. Retina. 2019;39: S74-83.

10. Meyer CH, Szurman P, Haritoglou C, et al. Application of subretinal fluid to close refractory full thickness macular holes: treatment strategies and primary outcome: APOSTEL study. Graefes Arch Clin Exp Ophthalmol. 2020;258(10):2151-61.

11. Ruiz-Moreno JM, Staicu C, Piñero DP, Montero J, Lugo F, Amat P. Optical coherence tomography predictive factors for macular hole surgery outcome. Br J Ophthalmol. 2008;92(5):640-4.

12. Wakely L, Rahman R, Stephenson J. A comparison of several methods of macular hole measurement using optical coherence tomography, and their value in predicting anatomical and visual outcomes. Br J Ophthalmol. 2012;96(7):1003-7.

13. Ip MS, Baker BJ, Duker JS, et al. Anatomical outcomes of surgery for idiopathic macular hole as determined by optical coherence tomography. Arch Ophthalmol. 2002;120:29-35.

14. Lytvynchuk LM, Falkner-Radler CI, Krepler K, et al. Dynamic intraoperative optical coherence tomography for inverted internal limiting membrane flap technique in large macular hole surgery. Graefes Arch Clin Exp Ophthalmol. 2019;257(8):1649-59.

15. Duker JS, Kaiser PK, Binder S, et al. The international vitreomacular traction study group classification of vitreomacular adhesion, traction and macular hole. Am Acad Ophthalmol. 2013;120: 2611-9.

16. Okanouchi T, Toshima S, Kimura S, Morizane Y, Shiraga F. Novel technique for subretinal injection using local removal of the internal limiting membrane. Retina. 2016;36:1035-8.

17. Wells JA, Gregor ZJ. Surgical treatment of fullthickness macular holes using autologous serum. Eye (Lond). 1996;10:593-9.

18. Hoerauf H, Kluter H, Joachimmeyer E, et al. Results of vitrectomy and the no-touch-technique using autologous adjuvants in macular hole treatment. Int Ophthalmol. 2001;24:151-9.
19. Michalewska Z, Michalewski J, DulczewskaCichecka K, Nawrocki J. Inverted internal limiting membrane flap technique for surgical repair of myopic macular holes. Retina. 2014;34:664-9.

20. Michalewska Z, Michalewski J, DulczewskaCichecka K, Nawrocki J. Temporal inverted internal limiting membrane flap technique versus classic inverted internal limiting membrane flap technique: a Comparative Study. Retina. 2015;35: 1844-50.

21. Boninska J, Nawrocki J, Michalewska Z. Mechanism of "flap closure" after the inverted internal limiting membrane flap technique. Retina. 2018;38(11): 2184-9.

22. Rizzo S, Belting C, Genovesi-Ebert F, Cresti F, Vento A, Martini R. Successful treatment of persistent macular holes using "heavy silicone oil" as intraocular tamponade. Retina. 2006;26:905-8.

23. Rizzo S, Tartaro R, Barca F, Caporossi T, Bacherini D, Giansanti F. Internal limiting membrane peeling versus inverted flap technique for treatment of fullthickness macular holes: a comparative study in a large series of patients. Retina. 2018;38(Suppl 1): S73-8.

24. Khodani M, Bansal P, Narayanan R, Chhablani J. Inverted internal limiting membrane flap technique for very large macular hole. Int J Ophthalmol. 2016;9:1230-2.

25. Al Sabti K, Kumar N, Azad RV. Extended internal limiting membrane peeling in the management of unusually large macular holes. Ophthalmic Surg Lasers Imaging. 2009;40:185-7.

26. Mahalingam P, Sambhav K. Surgical outcomes of inverted internal limiting membrane flap technique for large macular hole. Indian J Ophthalmol. 2013;61:601-3.

27. Shin MK, Park KH, Park SW, Bzon IS, Lee JE. Perfluoro-n-octane-assisted single-layered inverted internal limiting membrane flap technique for macular hole surgery. Retina. 2014;34:1905-10.

28. Andrew N, Chan WO, Tan M, Ebneter A, Gilhotra JS. Modification of the inverted internal limiting membrane flap technique for the treatment of chronic and large macular holes. Retina. 2016;36: 834-7.

29. Hillenkamp J, Kraus J, Framme C, et al. Retreatment of full-thickness macular hole: predictive value of optical coherence tomography. Br J Ophthalmol. 2007;91:1445-9.

30. Kusuhara S, Teraoka Escano MF, Fujii S, et al. Prediction of postoperative visual outcome based on 
hole configuration by optical coherence tomography in eyes with idiopathic macular holes. Am J Ophthalmol. 2004;138:709-16.

31. Ruiz-Moreno JM, Staicu C, Pinero DP, Montero J, Lugo F, Amat P. Optical coherence tomography predictive factors for macular hole surgery outcome. Br J Ophthalmol. 2008;92:640-4.

32. Alkabes M, Padilla L, Salinas C, et al. Assessment of OCT measurements as prognostic factors in myopic macular hole surgery without foveoschisis. Graefes Arch Clin Exp Ophthalmol. 2013;251(11):2521-7.

33. Szigiato A, Gilani F, Walsh M, Mandelcorn ED, Muni RH. Induction of macular detachment for the treatment of persistent or recurrent idiopatic macular holes. Retina. 2016;36:1694-8.

34. Wong R. Retina expansion technique for macular hole apposition report 2: efficacy, closure rate, and risks of a macular detachment technique to close large full-thickness macular holes. Retina. 2018;38(4):660-3.

35. Chakrabarti R, Roufail E. Posterior pole detachment technique for the management of full thickness macular hole. Adv Ophthalmol Vis Syst. 2015;2(5): 00058

36. Claes C. Internal repair of very large, myopic and recurrent macular holes by creation of a central retinal detachment and silicone oil tamponade. Retina. 2019;39(Suppl 1):S72-3.

37. Sasaki H, Shiono A, Kogo J, et al. Inverted internal limiting membrane flap technique as a useful procedure for macular hole-associated retinal detachment in highly myopic eyes. Eye (Lond). 2017;31(4):545-50.

38. Wakabayashi T, Ikuno Y, Shiraki N, Matsumura N, Sakaguchi H, Nishida K. Inverted internal limiting membrane insertion versus standard internal limiting membrane peeling for macular hole retinal detachment in high myopia: one-year study. Graefes Arch Clin Exp Ophthalmol. 2018;256(8): 1387-93.

39. Chen SN, Yang CM. Inverted internal limiting membrane insertion for macular hole-associated retinal detachment in high myopia. Am J Ophthalmol. 2016;162:99-106.

40. Yun C, Oh J, Hwang SY, Togloom A, Kim SW, Huh K. Morphologic characteristics of chronic macular hole on optical coherence tomography. Retina. 2012;32:2077-84.

41. Kumagai K, Ogino N, Furukawa M, Ooya R, Horie E. Early centripetal displacements of capillaries in macular region caused by internal limiting membrane peeling. Clin Ophthalmol. 2018;27(12): 755-63.

42. Shiono A, Kogo J, Sasaki H, et al. Hemi-temporal internal limiting membrane peeling is as effective and safe as conventional full peeling for macular hole surgery. Retina. 2019;39(9):1779-85.

43. Schawkat $M$, Valmaggia $C$, Lang $C$, et al. Influence of postoperative posture on macular slippage after macula-off retinal detachment: a randomized controlled trial. Ophthalmol Ther. 2019;8(4):519-25. 